\title{
ESTUDO DA FLUIDODINÂMICA DE UM LEITO DE JORRO APLICADO A UMA MISTURA DE CASCA DE COCO E AREIA VIA ANÁLISE EXPERIMENTAL E SIMULAÇÃO POR CFD
}

\author{
C. G. FERREIRA ${ }^{1}$, F. B. SANTOS ${ }^{1}$, K. M. BARCELOS ${ }^{2}$, D. C. RIBEIRO ${ }^{1,2}$ e T. S. LIRA ${ }^{1,2}$ \\ ${ }^{1}$ Universidade Federal do Espírito Santo, Departamento de Engenharias e Tecnologias \\ ${ }^{2}$ Universidade Federal do Espírito Santo, Programa de Pós-Graduação em Energia \\ E-mail para contato: taisa.lira@ufes.br
}

\begin{abstract}
RESUMO - A diminuição da disponibilidade dos recursos energéticos não renováveis, associado aos impactos ambientais causados pelo seu uso, ascendeu o número de pesquisa com fontes de energias renováveis. Durante o beneficiamento do coco verde, por exemplo, há a geração de resíduos que apresentam condições à transformação em biocombustível. A técnica de pirólise vem recebendo atenção e o leito de jorro é investigado como reator promissor para esse processo, devido ao bom contato gás-sólido que proporciona. Entretanto, a complexidade fluidodinâmica nesse equipamento permanece sendo o principal obstáculo para sua utilização. Assim, tem-se como objetivo investigar o comportamento fluidodinâmico das misturas de casca de coco e areia por meio de análises experimentais e simulação via CFD, a fim de compreender as limitações deste equipamento. Para avaliar o efeito da fração mássica da casca de coco $(10,25$ e 40 $\%$ ) e altura de leito estático $(6,8,10 \mathrm{~cm})$ sobre a condição de jorro mínimo, foi realizada um planejamento fatorial $3^{2}$ com dois pontos centrais. $\mathrm{O}$ modelo Euleriano Granular Multifásico foi utilizado para descrever o escoamento gássólido no leito de jorro e mostrou-se adequado para representar qualitativamente esse fenômeno.
\end{abstract}

\section{INTRODUÇÃO}

A evolução dos processos industriais e o desenvolvimento da sociedade acarretam em um aumento sistemático da demanda de energia. Associado a isto, tem-se a preocupação em se preservar o meio ambiente, tornando necessária a busca de alternativas energéticas sustentáveis. Assim, a biomassa surge como uma oportunidade viável ambiental e economicamente, visto que é abundante, de baixo custo e renovável.

O aproveitamento energético da biomassa em leito de jorro pode ser feito por intermédio da pirólise, já que este equipamento apresenta, segundo Santos (2011), excelente contato gás-partícula, altas taxas de transferência de calor e massa e alta taxa de recirculação de partículas. Os parâmetros fluidodinâmicos de maior importância no estudo do regime de jorros são: a velocidade mínima de jorro, a altura máxima de jorro estável e a perda de carga em função da velocidade do ar (MARRETO, 2006), os quais constituem o maior obstáculo para a utilização do leito de jorro devido a sua complexidade. Além disso o emprego do leito 
de jorro no processo de pirólise implica diretamente na adição de material particulado inerte, geralmente areia. Além de conferir estabilidade fluidodinâmica, as partículas de areia são responsáveis pela maior parte do aquecimento da biomassa (SANTOS, 2013).

Segundo Béttega (2009), as simulações numéricas utilizando a técnica de Fluidodinâmica Computacional (CFD) são importantes ferramentas para a investigação dos regimes de escoamento ar-sólido presentes no leito de jorro, uma vez que não há necessidade de inserção de sondas que poderiam interferir no escoamento, sendo possível investigar uma ampla faixa de condições operacionais, parâmetros geométricos e materiais com diferentes propriedades.

Trabalhos referentes à simulação CFD de partículas em leitos de jorro são encontrados na literatura. Duarte et al. (2005), por exemplo, descreveram a fluidodinâmica das partículas e do gás no interior de um leito de jorro usando uma Modelagem Euleriana Granular Multifásica. Seus resultados simulados foram comparados com dados experimentais obtidos em laboratório e com os resultados de HE et al. (1994a,b), demonstrando uma boa correlação entre ambos. Além disso, as informações acerca da fluidodinâmica das fases contínua e dispersa representaram um importante passo no desenvolvimento de geometrias ótimas deste equipamento.

Diante deste contexto, tem-se como objetivo investigar o comportamento da fluidodinâmica da areia e casca de coco, de forma a apontar condições operacionais de processo adequadas para o bom funcionamento do reator por meio de estudos experimentais e simulação via CFD.

\section{METODOLOGIA}

\subsection{Materiais}

O coco verde, pertencente a variedade Anão foi obtida por doação de estabelecimentos comerciais de São Mateus/ES. A casca do coco passou por um pré-tratamento que consistiu em lavagem com água, secagem em estufa por duas horas, sendo triturada em moinho de facas e por fim peneirada. A Tabela 1 apresenta os dados de referentes as propriedades físicas e químicas da casca de coco e da areia.

Tabela 1 - Propriedades químicas e físicas dos materiais.

\begin{tabular}{|c|c|c|c|}
\hline \multicolumn{4}{|c|}{ Propriedades químicas do coco } \\
\hline \multicolumn{3}{|c|}{ Composição lignocelulósica } & Análise imediata \\
\hline Extrativos (\%) & $8,12 \pm 0,14$ & Umidade (\%) & $8,49 \pm 0,15$ \\
\hline Lignina (\%) & $30,30 \pm 0,04$ & Voláteis (\%bs) & $82,35 \pm 0,44$ \\
\hline Hemicelulose (\%) & $29,68 \pm 0,49$ & Cinzas (\%bs) & $2,56 \pm 0,07$ \\
\hline Celulose (\%) & $30,32 \pm 0,02$ & Carbono fixo* (\%bs) & $15,09 \pm 0,51$ \\
\hline \multicolumn{3}{|c|}{ Propriedades físicas das partículas } \\
\hline Propriedade & Casca de coco & Areia \\
\hline \multicolumn{2}{|c|}{ Massa específica aparente $\left[\mathrm{kg} / \mathrm{m}^{3}\right]$} & $478,62 \pm 3,04$ & $2644,98 \pm 5,36$ \\
\hline Massa específica real [kg/m $\left.{ }^{3}\right]$ & $1539,50 \pm 1,20$ & - \\
\hline Diâmetro médio [mm] & $2,58 \pm 0,22$ & $1,55 \pm 0,15$ \\
\hline Esfericidade & $0,60 \pm 0,09$ & $0,79 \pm 0,05$ \\
\hline
\end{tabular}




\subsection{Ensaios Fluidodinâmicos}

Os ensaios fluidodinâmicos foram realizados em um leito de jorro cônico em escala piloto, instalado no Laboratório de Eficiência Energética I do PPGEN/UFES, utilizando amostras de coco verde e areia como material inerte.

Realizou-se um planejamento fatorial $3^{2}$ com dois pontos centrais, resultando em 11 experimentos, a fim de analisar os efeitos da composição da mistura, ou seja, fração mássica das partículas de casca de coco $(\mathrm{X})$ e altura de leito estático $(\mathrm{H})$ sobre a mínima velocidade de ar em que os materiais encontram-se jorrando $\left(\mathrm{V}_{\mathrm{mj}}\right)$ e sua respectiva queda de pressão $\left(-\Delta \mathrm{P}_{\mathrm{mj}}\right)$.

As amostras de cada material foram misturadas e adicionadas ao recipiente cônico até atingir a altura de leito estático. Em seguida, o ar foi injetado ao leito, de forma que sua velocidade fosse gradativamente aumentada até um valor $20 \%$ maior que a velocidade de jorro incipiente e, em seguida, reduzida à velocidade mínima alcançada pelo equipamento. A partir da realização desses experimentos e em posse dos pares de velocidade e queda de pressão no leito, por meio do programa de aquisição de dados Labview, as curvas características foram construídas.

\subsection{Simulação via CFD}

Foram construídas 5 malhas estruturadas, por meio do software ANSYS ICEM CFD, compostas por células quadriláteras, com eixo de simetria axial. Este procedimento é válido, já que o regime de jorro obtido experimentalmente foi estável, garantido a simetria do leito.

O teste de independência foi realizado utilizando as malhas com um mesmo modelo matemático a fim de verificar quando o refinamento da malha não trará alterações significativas nas características do escoamento. Usou-se uma mistura contendo $25 \%$ em massa de casca de coco, altura de leito estático de $8 \mathrm{~cm}$, velocidade do ar $20 \%$ acima da velocidade de mínimo jorro aplicando as condições de simulação descritas na Tabela 2. Os dados de diâmetro e massa especifica estão na Tabela 1.

Tabela 2 - Parâmetros com intuito de serem aplicados na simulação via CFD.

\begin{tabular}{|c|c|c|}
\hline Item Setup & Parâmetro & Descrição \\
\hline Solver & Pressure-based; Transiente; 2D axissimétrico & \\
\hline \multirow{2}{*}{ Modelos } & Modelo Euleriano Multifásico & 3 fases (ar, areia e casca de cacau) \\
\cline { 2 - 3 } & Modelos de turbulência & $\mathrm{k}-\varepsilon$ (Padrão disperso) \\
\hline \multirow{2}{*}{ Materiais } & Densidade do ar & $1,205 \mathrm{~kg} \mathrm{~m}^{-3}$ \\
\cline { 2 - 3 } & Viscosidade do ar & $1,82.10^{-5} \mathrm{~kg} \mathrm{~m}^{-1} \mathrm{~s}^{-1}$ \\
\hline $\begin{array}{c}\text { Condições } \\
\text { de } \\
\text { contorno }\end{array}$ & Entrada & Velocidade de entrada do gás com perfil plano \\
\cline { 2 - 3 } & Saída & Pressão atmosférica \\
\hline $\begin{array}{c}\text { Condição } \\
\text { inicial }\end{array}$ & Parede & 0,06 m;0,08 m;0 de não deslizamento para as fases \\
\cline { 2 - 3 } & Altura do leito estático & 0,$476 ; 0,485 ; 0,498$ \\
\hline
\end{tabular}

Utilizando o software ANSYS FLUENT v. 15.0.0 preferiu-se usar o Modelo Euleriano por ser mais complexo e ter a capacidade de resolve um conjunto de $\mathrm{n}$ equações de momento e continuidade para cada fase. O acoplamento é conseguido por meio da pressão e de coeficientes de troca entre as fases. Para fluxos granulares as propriedades são obtidas pela 
aplicação da teoria cinética granular. Este modelo é apropriado para leitos fluidizados e de jorro (ANSYS FLUENT THEORY GUIDE, 2013).

O modelo de Gidaspow prediz qualitativamente os perfis de porosidade e velocidade das partículas no interior do equipamento. O modelo $k-\varepsilon$ inclui duas equações, "k" (energia cinética turbulenta) representa a variação das flutuações da velocidade, e "ह" a taxa de

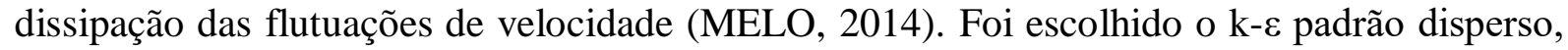
uma vez que o escoamento turbulento em leitos de jorro ocorre na região de jorro, caracterizada por uma baixa porosidade (ANSYS FLUENT THEORY GUIDE, 2013).

\section{RESULTADOS E DISCUSSÕES}

Segundo van Wachem (2000), o tamanho da malha deve ser determinado pelo refinamento até que as mudanças nas propriedades médias do sistema sejam menores que $4 \%$. A Figura 1a apresenta os resultados de velocidade do ar pela distância axial do leito de jorro em 5 malhas estruturadas: 1416, 2812, 8722, 10797 e 12272 células. Nota-se a influência do refinamento da malha, observa-se um desvio pequeno entre as malhas com 10797 e 12272 células.

Figura 1- (a) Teste de independência da malha e (b) Gráfico da queda de pressão em função do tempo de simulação.

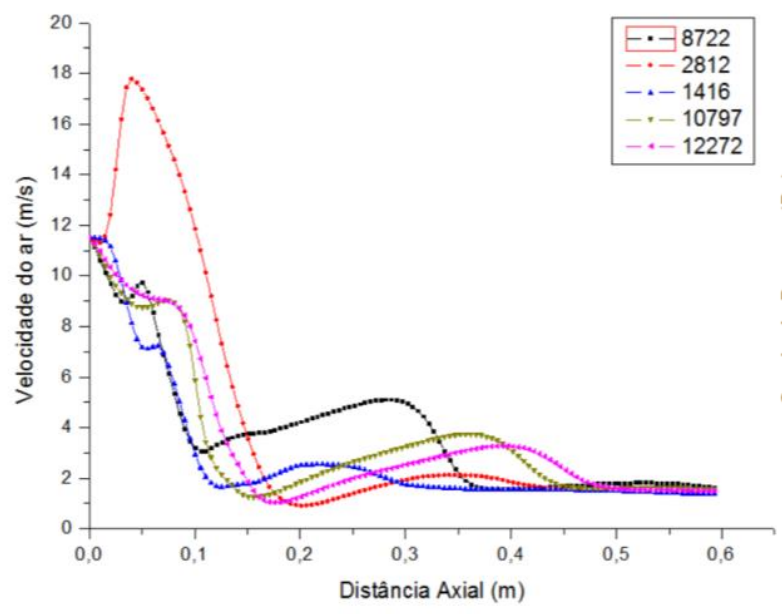

(a)

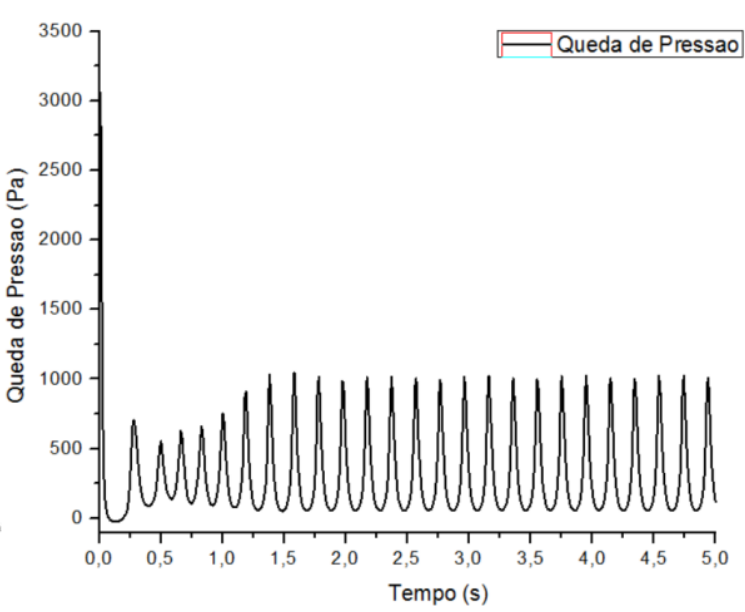

(b)

Para a malha de 10797 células a queda de pressão foi de 308,19 Pa enquanto a contendo 12272 foi de $309,74 \mathrm{~Pa}$ o que representa um erro em relação a malha anterior de $0,50 \%$. Assim, a malha com 10797 células foi escolhida para as demais simulações por presentar um tempo de simulação menor quando comparada à malha de 12272 células. As simulações foram realizadas em 5 segundos, baseando-se no trabalho de Santos (2011). Por meio do gráfico da queda de pressão em função do tempo de simulação (Figura 1b), verifica-se que esse tempo foi suficiente para alcançar o estado estacionário e que nos últimos segundos simulados, as propriedades médias do sistema não se alteram significativamente. Portanto, deve ser usado para caracterizar o sistema.

Para as mesmas condições empregadas no teste de malha, os perfis da fração volumétrica dos sólidos foram obtidos e podem ser observados na Figura 2. Verifica-se que a 
modelagem proposta é adequada para descrever o comportamento qualitativo do leito de jorro preenchido com misturas de casca de coco e areia, com as três regiões características do equipamento, região anular, de jorro e fonte bem evidenciadas nos perfis.

Figura 2 - Contorno da fração volumétrica da (a) areia, (b) casca de coco e (c) ar no ponto central.

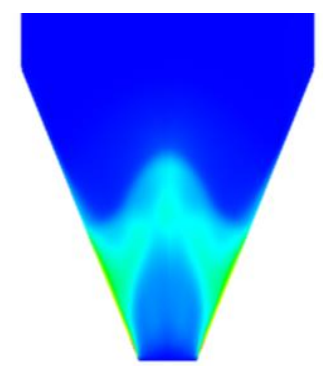

(a)

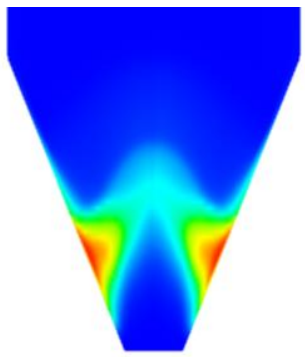

(b)

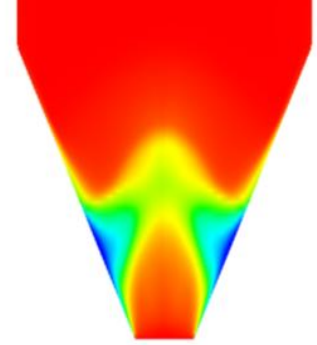

(c)

A Tabela 3 apresenta as quedas de pressão experimentais e simuladas via CFD para misturas de areia e casca de coco em diferentes alturas e frações mássicas de biomassa.

Tabela 3 - Comparação das quedas de pressão simuladas com a experimental.

\begin{tabular}{|c|c|c|c|c|c|c|c|c|}
\hline Teste & $\mathrm{X}$ & $\mathrm{H}(\mathrm{cm})$ & $\mathrm{X}_{1}$ & $\mathrm{X}_{2}$ & $\begin{array}{c}\mathrm{V}_{\mathrm{mj}} \\
(\mathrm{m} / \mathrm{s})\end{array}$ & $\begin{array}{c}-\Delta \mathrm{P}_{\mathrm{mj}} \text { Experimental } \\
(\mathrm{Pa})\end{array}$ & $\begin{array}{c}-\Delta \mathrm{P}_{\mathrm{mj}} \text { Simulado } \\
(\mathrm{Pa})\end{array}$ & Desvio (\%) \\
\hline 1 & 0,10 & 6 & -1 & -1 & 6,97 & $487 \pm 28$ & 417 & $-14,20$ \\
\hline 2 & 0,10 & 8 & -1 & 0 & 8,71 & $620 \pm 46$ & 530 & $-14,42$ \\
\hline 3 & 0,10 & 10 & -1 & 1 & 9,87 & $725 \pm 57$ & 699 & $-3,54$ \\
\hline 4 & 0,25 & 6 & 0 & -1 & 6,97 & $319 \pm 23$ & 257 & $-19,43$ \\
\hline 5 & 0,25 & 8 & 0 & 0 & 9,58 & $421 \pm 31$ & 309 & $-26,70$ \\
\hline 6 & 0,25 & 10 & 0 & 1 & 11,03 & $484 \pm 44$ & 397 & $-18,10$ \\
\hline 7 & 0,40 & 6 & 1 & -1 & 6,10 & $253 \pm 25$ & 216 & $-14,78$ \\
\hline 8 & 0,40 & 8 & 1 & 0 & 8,71 & $333 \pm 36$ & 262 & $-21,42$ \\
\hline 9 & 0,40 & 10 & 1 & 1 & 11,03 & $394 \pm 33$ & 311 & $-21,00$ \\
\hline 10 & 0,25 & 8 & 0 & 0 & 9,58 & $424 \pm 35$ & 310 & $-26,92$ \\
\hline 11 & 0,25 & 8 & 0 & 0 & 9,87 & $422 \pm 38$ & 306 & $-27,55$ \\
\hline
\end{tabular}

Diante dos resultados obtidos, percebe-se que os valores simulados são subestimados em todas as condições trabalhadas, sendo a maior discrepância observada com o aumento da fração mássica da biomassa. Vale ressaltar, porém, ao analisar o desvio padrão das medidas experimentais, que os dados estão próximos. O possível motivo para ter ocorrido tal diferença se deve na concepção do projeto simulado no qual admitisse que as partículas são esféricas e sem irregularidades. Não contabilizar esses efeitos na modelagem pode afetar de forma negativa as interações sólido-fluido e tensões da fase granular, as quais influenciam nas características dos fluxos de campo, como distribuição de sólidos e queda de pressão (DECKER et al., 2004).

\section{CONCLUSÕES}

Misturas que apresentam frações mássicas iguais ou inferiores a 40\% de biomassa são adequadas para proceder a pirólise, uma vez que regimes de jorro estáveis foram alcançados em todas as condições experimentais trabalhadas. Com relação à altura de leito estático, sugere-se que a técnica de pirólise seja realizada com altura de $10 \mathrm{~cm}$, por possuir maior 
quantidade de biomassa a ser processada e convertida em energia. $\mathrm{O}$ modelo Euleriano Granular Multifásico se mostrou adequado para representar qualitativamente o comportamento da mistura de areia e casca de cacau nas alturas e frações mássicas propostas.

\section{REFERÊNCIAS}

ANSYS FLUENT THEORY GUIDE. ANSYS, Inc., Canonsburg, PA, 2013.

BÉTTEGA, R. Contribuições ao estudo da fluidodinâmica em leito de jorro utilizando-se CFD. Tese (Doutorado em Engenharia Química) - Programa de Pós-Graduação em Engenharia Química, Universidade Federal de São Carlos, São Carlos, 2009.

DECKER, R. K.; MEIER, H. F.; MORI, M. Validação de modelo tridimensional para o escoamento gás-sólido diluído vertical e horizontal, por técnicas de fluidodinâmica computacional (CFD). Boletim Técnico Petrobras, Rio de Janeiro. v. 47, p. 88-100, 2004.

DUARTE, C. R.; MURATA, V. V. e BARROZO, M. A. S. A Study of the Fluid Dynamics of the Spouted Bed Using CFD, Brazilian Journal of Chemical Engineering, Vol. 22, p. 263-270, 2005.

HE Y. L.; LIM C. J.; GRACE J. R.; ZHU J. X.; QIN S. Z. Measurements of Voidage Profiles in Spouted Beds. Canadian Journal of Chemical Engineering, Vol. 72, p. 229-234, 1994a.

HE, Y. L.; QIN, S. Z.; LIM, C. J.; GRACE, J. R. Particle Velocity Profiles and Solid Flow Patterns in Spouted Beds. Can. J. Chem. Eng., Vol. 72, p. 561-568, $1994 \mathrm{~b}$.

MARRETO, R. N.Estudo da técnica de leito de jorro convencional para secagem de microcápsulas de caseína e pectina. Dissertação (Doutorado em Ciências Farmacêuticas) - Programa de Pós-graduação em Ciências Farmacêuticas, Universidade de São Paulo, Ribeirão Preto, 2006.

MELO, J. L. Z. Análise do leito de jorro como sistema de contato para pirólise de compósito PEBD/AL. 2014. 72 f. Dissertação (Mestrado em Energia) - Programa de Pós-Graduação em Energia, Universidade Federal do Espírito Santo, São Mateus, 2014.

SANTOS, K. G. Aspectos fundamentais da pirólise de biomassa em leito de jorro: fluidodinâmica e cinética do processo, 2011. Tese (Doutorado em Engenharia Química) Programa de Pós-Graduação em Engenharia Química, Universidade Federal de Uberlândia, Uberlândia, 2011.

SANTOS, N. A. V. Pirólise rápida de coprodutos do processo produtivo do biodiesel: efeito das condições de pirólise e caracterização dos produtos. 2013. Dissertação (Mestrado em Agroquímica) - Programa de Pós-Graduação em Agroquímica, Universidade Federal de Lavras, Lavras, 2013.

VAN WACHEM, B. G. M. Derivation, implementation, and validation of computer simulation models for gas-solid fluidized beds. Dissertação, Delf University of Technology, 2000. 